\title{
The Relevance of a Choice of Auction Format in a Competitive Environment
}

\author{
MATTHEW O. JACKSON \\ California Institute of Technology \\ and \\ ILAN KREMER \\ Stanford University
}

First version received July 2004; final version accepted January 2006 (Eds.)

\begin{abstract}
We examine the relevance of an auction format in a competitive environment by comparing uniform and discriminatory price auctions with many bidders in a private values setting. We show that if the number of objects for sale is small relative to the number of bidders, then all equilibria of both auctions are approximately efficient and lead to approximately the same revenue. When the number of objects for sale is proportional to the number of bidders, then the particulars of the auction format matter. All equilibria of the uniform auction are efficient, while all of the equilibria of the discriminatory auction are inefficient. The relative revenue rankings of the auction formats can go in either direction, depending on the specifics of the environment. These conclusions regarding the efficiency and revenue ranking are in contrast to the previous literature, which focused on the case of independent information across agents.
\end{abstract}

\section{INTRODUCTION}

In many markets, including treasury auctions, Initial Public Offerings, security markets, and internet-based markets, large numbers of agents compete for a limited supply of resources. These markets use a variety of rules for setting prices, including uniform price auctions, discriminatory price (pay-your-bid) auctions, and hybrids of these, among others. A fundamental question arises as to when competitive pressures render the choice of mechanism irrelevant.

A classic view is that with many agents, a set supply of objects (either fixed or in per capita terms) and some reasonable trading mechanism, an equilibrium of most reasonable auction formats should approach a competitive equilibrium, and so the particulars of the trading mechanism should be largely irrelevant. In the limit, the agents who value the objects the most should obtain them and end up paying a price that is close to the marginal valuation-that is, where the demand and supply curves cross. ${ }^{1}$

In this paper we examine and refute this view by comparing the two canonical auction formats: a uniform price auction where all units are sold at the same price, which is some variation

1. An important debate regards the structure of Treasury auctions. For, the most part this literature does not consider the effect of a high degree of competition, which is the main focus of this paper. For some of the highlights of that literature see Friedman (1960), Bikhchandani and Huang (1989), Chari and Weber (1992), Back and Zender (1993), Nyborg and Sundaresan (1996), Hortasçu (2001), and Klemperer (2003). 
of the highest non-winning bid, ${ }^{2}$ and a discriminatory auction where the winning bids are the prices paid. Our main result is that there are no differences between auction formats if and only if the number of items being sold is negligible compared to the number of bidders. If the supply is non-negligible, then the auction format has implications with regards to both efficiency and expected revenues.

To be more specific, we consider the following setting. A seller is interested in selling some number of identical objects to a number of potential buyers. We examine limits as the number of buyers (and sometimes the number of objects) becomes large. Each buyer may be interested in one or perhaps more units. Our interest is in comparing the two most prominent auction formats: a uniform price auction and a discriminatory auction. We consider the resulting allocations and the expected revenues the seller obtains. In both mechanisms, the supply is awarded to the highest $k$ bids. The difference lies in the way prices are set. This turns out to have important implications for equilibrium bidding behaviour and the efficiency of the allocation of the goods.

We first consider situations where the supply of objects is fixed and thus small relative to the number of bidders as we let the number of bidders grow. There we find that

1. If the supply is fixed then the allocations of all equilibria of either auction format converge to efficient allocations and the seller extracts the full surplus (i.e. the seller's revenue converges to the sum of the winning buyers' valuations). ${ }^{3}$ We illustrate this in Section 2 in Example 1 and show it formally in Section 4.

Next, we consider the case where the supply of objects grows in proportion to the number of bidders. There we find that

2. If the number of units is proportional to the number of bidders then the auction format matters and impacts both the allocation and the expected revenues.

- In terms of efficiency, all equilibria of the uniform price auction converge to efficient allocations; while with multiple-unit demands and decreasing marginal valuations, all equilibria of the discriminatory auction lead to inefficient allocations, even in the limit. We illustrate this in Section 2 in Example 2 and show it formally in Section 5.1.

- In terms of revenue, the ranking is ambiguous (despite the clear ranking in terms of efficiency).

- In the case where agents desire only a single unit (or multiple units at the same marginal valuation), the auctions can be clearly ranked and the uniform price auction yields strictly higher limiting revenues than the discriminatory auction. We illustrate this in Section 2 in Example 3 and show it formally in Section 5.3.

- In cases where agents desire more than one unit and have decreasing marginal valuations for additional units, then the ranking can go either way, and in some cases the discriminatory auction can yield strictly higher limiting revenues than the uniform price auction. We illustrate this in Section 2 in Example 4.

Understanding the difference between a fixed supply and a proportional supply of objects for sale helps develop intuition for our results.

2. There are some variations to consider here, depending on whether agents pay only the highest non-winning bid among the other agents or among all agents (so one of their own bids might set the price). All such variations lead to the same consequences from our perspective.

3. In the limit, with a fixed number of objects and a large number of bidders, competitive pressures ensure that the winning bidders will all have nearly the same value and bid close to their values. So, there would be vanishing consumer surplus in a competitive market. Thus, "full surplus extraction" refers to the fact that the price converges to the competitive price for that case and is not a deep statement about extraction of consumer surplus. 
When there is a fixed supply of units for sale (that is small relative to the number of bidders), an agent realizes that when he or she wins an object there are necessarily other bidders who have very similar information and valuations who are not getting objects. Regardless of the mechanism, this yields a competitive pressure that pushes bidders to bid close to their values in order to have any chance of winning and implies that only the bidders with the very highest types will get objects. This implies an efficient allocation and results in the full possible revenue going to the seller.

In contrast, when the supply of objects is proportional to the number of bidders, then even with many bidders, and many of each type, ex post there will be whole neighbourhoods of types who end up getting objects and whole neighbourhoods of other types who do not. Changing a bid now leads to a continuous change in the expectation of obtaining an object. Even though an individual bidder is negligible in terms of the overall allocation, each bidder realizes that adjusting his or her bid provides important trade-offs between the price that he or she pays and the probability of obtaining an object. Here there is an opportunity for the mechanism to matter, and indeed it does.

With a uniform price auction, agents are essentially price takers as changing their bid can have little impact on the price, and so they have incentives to bid their value as that means that they win under precisely the right circumstances (when the price is below their value). This leads to efficient allocations, even with multiple-unit demands.

However, in a discriminatory price auction agents are not price takers-even in the limit. Their bids affect their payments and they have an incentive to lower their bids to lower the price that they pay, and they trade this off against the probability of no longer winning an object. If each agent desires at most one unit, then under affiliated values, agents with higher values have higher expectations about where the marginal bid will lie, and hence they bid more aggressively. Thus, bids turn out to be monotone in value and the allocation is efficient. However, if agents demand more than one unit and have decreasing marginal valuations, then it is possible that agents who have the same value for some object (say the first object for one bidder and the second for another bidder) could have very different beliefs about the aggregate demand. This breaks the monotonicity of bidding, as it leads to differences in expectations that bidders with the identical value for an object have about the change in probability of winning due to a change in bids. It is similar to having agents see two separate pieces of information: one is their own value, and another is information about the aggregate demand. In such a world with an uncoupling of valuations and information about aggregate demand one would end up with inefficient allocations, and that is what is happening here. While in our model we have only a single signal per bidder, the fact that bidders have downward-sloping demands has a similar effect. ${ }^{4}$

The comparison of revenues between the discriminatory and uniform price auctions with a proportional supply of objects comes down to the incentives that bidders have to shade their bids on different objects. With single-unit demands, bidders shade bids sufficiently in a discriminatory auction so that there is an unambiguously lower revenue than in the uniform price auction. However, with multiple-unit demands, agents can end up bidding aggressively enough on some units that they end up paying more in a discriminatory auction than in a uniform auction. This depends on the particulars of the circumstances.

Before turning to the formal presentation of the model we make two further comments. Since we consider general multi-unit demands, there are some implicit asymmetries in the auctions we analyse. That is, bids for a first object of value $v$ can be very different from bids on

4. This is different from the inefficiency noted by Ausubel and Cramton (2002), due to the monopsony power of large buyers. Such market power is not present in our model due to the large number of bidders. The intuition behind the inefficiency here is closer in spirit to that behind asymmetric auctions as in Maskin and Riley (2000), where agents with similar values can end up with different beliefs. Here, it arises in an initially symmetric world and in large auctions. 
a second object of value $v$, because the bidders have different information about which price is likely to clear markets. This is at the heart of the discussion above, is illustrated in Example 2 below, and is the critical factor in understanding the properties of and differences between the auction formats. Unfortunately, this also makes equilibria a challenge to characterize. Nevertheless, while we cannot always obtain closed forms for the equilibria, we can still outline the efficiency properties and, in some cases, we can make comparisons of the revenues. In particular, in many cases our approach applies to all equilibria of these auction formats, including asymmetric equilibria and/or those in mixed strategies.

It is also important to emphasize that a number of our results do not require any affiliation of valuations. It is only when we need to examine the equilibria of discriminatory auctions under a proportional supply of objects that such conditions become important in deducing equilibrium behaviour.

The rest of the paper is organized as follows. In Section 2 we illustrate the main results and intuition through several examples. We formalize and generalize these examples in the following sections. In Section 3 we describe the model. Section 4 examines the case of fixed total supply, Section 5 examines the case of a proportional supply (fixed per capita supply), and Section 6 discusses extensions such as multi-dimensional information and asymmetries and concludes.

\subsection{Contributions and relation to the literature}

Bulow and Klemperer (1996) show that increasing the number of bidders in an auction can lead to larger increases in revenue than using an optimal reserve price. This suggests that, in a sense, an increase in the number of agents can be more important than particulars of the auction design. Knowing this, one might be tempted to conjecture (as we initially did) that with large numbers of agents we do not have to worry about the particulars of auction design and reasonable auction formats should have the same properties. That is, one might conjecture that the mechanism design is secondary to the number of bidders in determining the outcome. Our results show that while this turns out to be true if the supply of objects is small relative to the number of bidders, it is not true if the supply is proportional to the number of bidders.

Bali and Jackson (2002) and Kremer (2002) consider auctions with growing numbers of bidders and a single unit of a good for sale. Kremer shows that in some common values settings the expected revenues of first price, second price, and English auctions all converge to the expected value of the object. ${ }^{5}$ Bali and Jackson show that such convergence holds in a wide class of auctions and information settings. The results in this paper generalize some of the above results to a case of multi-unit demand. Again, however, a critical insight here is that such equivalency results only hold when the supply of objects is negligible relative to the population size.

Swinkels $(1999,2001)$ examines a setting where the supply of objects is proportional to the number of bidders and bidders may have multi-unit demands. Swinkels shows that both the uniform and discriminatory auctions are efficient in the limit and also gets an asymptotic revenue equivalence result. While our conclusions regarding the uniform auction show that the uniform price auction is still efficient with correlation in valuations, our conclusion that the discriminatory auction can yield allocations that are largely inefficient, even in the limit, and that the revenue can differ substantially across auction formats, are both in contrast to his conclusions. The difference is that in Swinkels the valuations are independently distributed across bidders, while we allow for correlation in valuations. ${ }^{6}$ Under correlation, units that are valued similarly can have different

5. See Goeree and Offerman (1999) for a related result.

6. We have independent participation decisions across bidders, following Swinkels, as a tool to ensure uniqueness in equilibria. The difference of allowing for correlation in information is the key to the differences in conclusions about efficiency and revenue. 
information associated with them, which leads to different bidding behaviour and inefficient allocations under the discriminatory auction format.

\section{ILLUSTRATIONS OF THE MAIN RESULTS}

To get a better idea of some of the issues, let us consider a few simple examples that preview our central findings. We keep this discussion heuristic. Nevertheless, the reasoning behind our main results can be seen in these examples. For more detailed arguments see the theorems and proofs that follow.

\section{Example 1. A Small Number of Objects-Efficient and Equivalent Auctions}

Consider an auction with $n$ bidders. There is a number of objects for sale and to fix ideas let us say that these are tickets for a NBA game. To keep this example simple, let us suppose that each bidder wants to buy at most two tickets. Bidders vary in how much they are willing to pay, but there is some correlation among these values. This might represent some overall measure of fans' excitement, or how well the team is doing, and how important the particular game is. In particular, each bidder has a value $v_{i}$ for a first ticket and a value of $\frac{v_{i}}{2}$ for a second ticket. The valuations $v_{i}$ are independently and identically uniformly distributed between $X$ and some value $X+1$. Let $X$ be distributed uniformly on $[0,1]$. So, while the bidders' valuations are independent conditional on $X$, they are correlated when viewed unconditionally.

First, let us consider a case where there is some small number of tickets for sale, say 100 , and a relatively large number of bidders, say $n=10,000$. With such a number of bidders, at most one out of 100 bidders will even obtain a single ticket, much less two. Moreover, each bidder knows that there are likely to be more than a hundred other bidders who have a valuation for buying one ticket within $1 / 100$ of their valuation. This competitive pressure leads bidders to bid nearly their value, and so in both the uniform or discriminatory auctions the objects are sold to agents who have valuations for the tickets that are nearly $X+1$ (the upper bound on valuations, whatever it turns out to be) and for prices of nearly $X+1$. This is efficient and leads to nearly the maximum possible revenue for the seller.

\section{Example 2. Proportional Numbers of Objects-Contrasts in Efficiency}

Next, consider the same information and valuation structure, but a case where the number of tickets for sale is proportional to the number of bidders. In particular, let us suppose that the number of tickets for sale is actually two-thirds the number of bidders. Remember that each bidder desires two tickets, and so there are three times as many tickets demanded as supplied. Things here are a bit more complicated to analyse, but nonetheless we can still reach some firm conclusions.

First, consider the uniform price auction, which is the easier of the two to analyse. With a large number of bidders, the chance that any given bidder's bid sets the price is negligible (see Lemma 1 below). Thus, bidders are effectively price takers. This means that bidders have incentives to bid at or near their valuations. Their bids play a large role in determining whether they get an object, but a negligible role in affecting the price. This leads to an approximately efficient allocation. In this case, the market clearing price is approximately $p=\frac{2}{3} X+\frac{4}{9}$, which would be the "competitive price". 7

7. Given the cut-off $p$, we know that agents with a signal between $p$ and $2 p$ get one unit while agents with a signal between $2 p$ and $X+1$ receive two units. Market clearing implies that $p+2(X+1-2 p)=2 / 3$, which implies that $p=\frac{2}{3} X+\frac{4}{9}$. 
Second, consider the discriminatory price auction. Let us argue that every equilibrium in a discriminatory auction will be significantly inefficient. Suppose, to the contrary, that there was an (approximately) efficient equilibrium. Consider a bidder with a valuation of a first ticket of $v_{i}=4 / 3$, and hence a valuation for a second ticket of $2 / 3$. Such a bidder knows that $X$ is at least $1 / 3$, and so the efficient cut-off value is at least $2 / 3$. As other bidders with values near $v_{i}=4 / 3$ have the same information-such a bidder's bid on the second unit will have to be close to $2 / 3 .{ }^{8}$ Now, consider a bidder with a valuation of a bit above $2 / 3$ for a first unit. Such a bidder only knows that $X$ lies somewhere between 0 and his value. Such a bidder assigns non-negligible probability that $X$ could be near 0 , which would lead to a cut-off value of $p$ near $4 / 9$. Such a bidder should clearly bid some significant amount less than $2 / 3$, as from that bidder's point of view, there is a significant probability that the market clearing price will be well below $2 / 3$. However, we have now reached a contradiction of efficiency: the bidders with $v_{i}$ 's close to $4 / 3$ are bidding near their value, $2 / 3$, for their second unit, and will outbid large numbers of bidders with $v_{i}$ 's above $2 / 3$, but who will bid well below $2 / 3$. We conclude that we get different limiting efficiency properties, with the uniform auction being nearly efficient and the discriminatory auction not so.

\section{Example 3. Proportional Numbers of Objects-Contrasts in Revenues I}

Let us now compare the revenue generated by the different auction formats.

The inefficient outcome in Example 2 results from the multiple demands of agents, which led to an uncoupling of agents' information and their valuations. In the context of discriminatory auctions, this leads to inefficiency, while in the context of uniform auctions it does not, and hence the contrast between the auctions.

When we turn to revenue comparisons, there are differences between the auctions, regardless of whether there is such an uncoupling of agents' information.

Let us first examine the case with a large number of bidders, but where bidders are only interested in buying a single object. Let there be fewer objects than bidders to keep things interesting. Here, there are equilibria of both auctions that lead to approximately efficient outcomes. ${ }^{9}$

Despite the fact that both auctions now yield the same allocation, they do not lead to the same revenues. In this case of unitary demands, the uniform price auction yields strictly higher revenues than the discriminatory auction, even in the limit.

To see this, suppose that there are only half as many tickets as bidders. Let us consider a slightly different information structure that makes some explicit calculations of revenue a bit easier. Each agent's value for an object is $v_{i}$, which is uniformly distributed on $[0, X]$, and $X$ is uniformly distributed on $[0,1] .^{10}$

We begin by analysing the discriminatory price auction format. The expression for the limit of the unique symmetric and monotone equilibrium bidding function, $b$, can be deduced from Milgrom and Weber (1982) (and omitted details from this example appear in the Appendix). It is

$$
b(v)= \begin{cases}\frac{1}{1+\ln (2)} v & \text { for } v<0.5 \\ \frac{1}{1+\ln (2)} 0.5 & \text { for } v \geq 0.5\end{cases}
$$

8. Under the supposition of efficiency, it must be that bidders with $v_{i}$ 's just below $4 / 3$ do not get objects, even though they have almost the same information and know that they are almost certain not to get a second object. If the bids on second objects were significantly below $2 / 3$, then such bidders could increase their bids and get objects with a non-trivial probability and at a price below value, which would lead to contradiction.

9. This can be deduced from an extension of Milgrom and Weber (1982).

10. See Jackson and Kremer (2006) for a similar example, but with completely common values. 
Conditional on $X=x$, we can then compute expected revenues by computing the average bid over winning signals. As for large $n$, the winning signals will approximately be uniformly distributed between $x / 2$ and $x$, and so limiting revenues conditional on $x$ either converge to $\frac{1}{1+\ln (2)}\left(\frac{3 x}{4}\right)$ or something strictly less than this in the case where $x>0 \cdot 5$. Then straightforward calculations lead to limiting expected revenues of $\frac{0.3225}{1+\ln (2)}$ or approximately $0 \cdot 19$.

Let us compare this to the revenue from a uniform price auction. In the unique symmetric equilibrium players use their dominant strategies of bidding $v_{i}$. This implies that the price is the valuation of the agent who has the $\frac{n}{2}$-th highest valuation. The price in a uniform auction thus converges to $\frac{x}{2}$ as $n \rightarrow \infty$. Taking expectations over $x$, we find that in the uniform auction the expected revenue per unit converges to $0 \cdot 25$.

Thus, the revenue in large enough uniform price auctions is strictly larger than that of discriminatory auctions. ${ }^{11}$

While this ranking of auctions extends beyond this example to all situations with single-unit demands, the revenue ranking may go either way with multi-unit demands. The following example provides a case where the discriminatory auction leads to higher revenue than the uniform auction.

\section{Example 4. Proportional Numbers of Objects-Contrasts in Revenues II}

Reconsider the setting of Example 3 with the following modifications. Agents now value two objects, with a value for the first object of $K+v_{i}$ and a value for the second object of $v_{i}$, where $K>1$ is defined below. There are $3 n / 2$ objects for sale. So, the efficient allocation is that each bidder gets at least one object, and the $n / 2$ bidders having the highest $v_{i}$ 's each get two objects.

The asymptotic expected revenue per object in the Vickrey auction is easy: it converges to $X / 2$ and in expectation is $0 \cdot 25$.

In the discriminatory auction, the following is the limit of a sequence of equilibria for large enough $K$ : bidders' first bid is always $0.5 /(1+\ln (2))$; and the bidders' second bid is as described in Example 3. Without providing full detail, let us sketch why this is the limit of a sequence of equilibria. If all the first bids are above the support of the low bids, then the second bids are still the limit of a sequence of equilibria since the $n$ bidders end up bidding for the remaining $n / 2$ items, exactly as in Example 3. So the argument is that for some large enough $K$ and $n$, all bidders place their first bid at the top of the support of the lower bids. This simply requires that when looking conditional on any $v_{i}$, there is some minimal (bounded away from 0 ) conditional density of the cut-off bid falling near the top of the support (which must happen in an equilibrium). Even if a bidder sees a low $v_{i}$, that bidder still places some chance on high $X$ 's. Then for large $K$, lowering a bid by some $\varepsilon$ below the top of the support of the expected cut-off will lower the payment by $\varepsilon$ when winning, but loses a value of at least $K$ with probability $\varepsilon$ times the marginal probability lost, which is bounded below.

This sequence of equilibria is asymptotically efficient ${ }^{12}$ and also gives higher revenue in the limit than the Vickrey (uniform price) auction. The revenue is as follows: in the limit $2 / 3$ of the objects are sold at a price of $1 / 3$, and $1 / 3$ of them are sold at a price of slightly more than $0 \cdot 19$; so this is an average price of more than $0 \cdot 26$, which is higher than average price in the Vickrey auction of $0 \cdot 25$.

11. Also note that both mechanisms fail to extract the full surplus from the bidders even in the limit. The expected average value of the goods to winning bidders is $E\left[\frac{3 X}{4}\right]=0.325$. This is a more general phenomenon that is discussed in Jackson and Kremer (2004).

12. The example can be modified to be inefficient, and so the ranking of revenues has no general relationship with asymptotic efficiency. 
This example shows, when combined with Example 3, that one cannot generally rank the auctions in terms of the asymptotic revenue they generate. Here, the downward-sloping demands introduce large enough additional asymmetries between first and second object valuations to result in some interesting behaviour and reversal in revenue ranking from what we saw before.

This example also provides a comment on Example 2 regarding the inefficiency of the discriminatory auctions. Here $K$ is large enough, so that first objects are effectively not competitive with second objects. It is almost as if there are two separate auctions going on, and this results in efficient allocations.

\section{THE MODEL}

\subsection{Economies}

We examine sequences of economies with increasing numbers of agents. A sequence of economies is indexed by $n$, the number of agents in the economy. The agents are potential buyers of objects in an auction.

A supply of $k_{n}$ identical units of a good is to be sold in economy $n$.

Each agent values up to $m \geq 1$ units of the good.

We examine the two canonical cases of interest: ${ }^{13}$

- If $k_{n}=k$ for some $k$ and all $n$, then we say that there is a fixed supply of objects.

- If there exists $b \in(0, m)$ such that $\frac{k_{n}}{n} \rightarrow b$, then we say that there is a proportional supply of objects.

Many standard auction analyses with a single unit for sale, clearly fall into the fixed case, while analyses of auctions such as treasury auctions fall into the proportional case. Here, in the proportional supply case, we span between the extreme cases where $b=0$, which is essentially the fixed supply case, and $b=m$, which would be a per capita supply that would completely satiate all agents.

\subsection{Information}

Information is described by a framework that we borrow from Milgrom (1981), as described in detail below. This information structure is particularly suited to the discussion of growing sequences of economies, all based on the same underlying information structure. We use upper case letters to denote random variables and lower case letters to denote realizations. We use $f$ to denote a density or conditional density of a random variable and $F$ to denote a distribution. In some cases when it may be unclear to which random variables we refer, we use subscripts such as $f_{X}(\cdot)$, while when it is clear we will omit the subscripts.

Each agent $i \in\{1, \ldots, n\}$ in economy $n$ observes a private signal $S_{i}$ that takes on values in $[0,1]$. There is also an underlying random variable $X$ taking on values in $[0,1]$. The $S_{i}$ 's are independently and identically distributed conditional on $X$. This conditional distribution of $S_{i}$ given $X$ is described by a density function $f\left(s_{i} \mid x\right)$.

Take $X$ to be distributed on $[0,1]$ with a continuous density with connected support.

This information structure is symmetric across agents and uni-dimensional. We discuss allowing for asymmetries and multi-dimensional information in Section 6. The assumption that the random variables have continuous distributions is made to simplify the exposition, but is not critical to the results.

13. We chose these two categories to simplify the exposition. A tighter classification is whether the supply per capita converges to 0 . 
Let $S$ denote the vector of signals $S_{1}, \ldots, S_{n}$, and let $S_{-i}$ denote the vector of signals omitting $S_{i}$. Let $Y(k)$ denote the $k$-th order statistic of the signals $S$ and let $Y_{-i}(k)$ denote the $k$-th order statistic of the signals $S_{-i}$.

We assume that

Assumption 1. The unconditional (marginal) density of each $S_{i}, f\left(s_{i}\right)$, is positive for all $s_{i} \in[0,1]$. For the conditional distribution, there exists $\alpha>0$ such that $f(x)>0$ implies that $f_{S_{i} \mid X}(\cdot \mid x)$ has connected support and $f\left(s_{i} \mid x\right) \geq \alpha$ for all $s_{i}$ in that support.

Assumption 1 is a condition that guarantees that in a large enough economy, any agent (regardless of what signal they have observed) expects that there will be many other agents who will have observed arbitrarily nearby signals. This condition provides for competitive pressures in the limit.

\subsection{Preferences}

The value of the $j$-th unit for an agent who has a signal $s_{i}$ is given by $v_{j}\left(s_{i}\right)$. We assume that

Assumption 2. Agents have private values and desire up to $m \geq 1$ units. Their nonincreasing marginal utilities for additional units are described by $\left\{v_{j}\left(s_{i}\right)\right\}_{j=1}^{m}$, where $v_{j}\left(s_{i}\right) \geq$ $v_{j^{\prime}}\left(s_{i}\right)$ for any $s_{i}$ and $j \leq j^{\prime}$. Each $v_{j}$ is differentiable and increasing in $s_{i}$. We normalize $v_{1}\left(s_{i}\right)$ to be equal to $s_{i}$.

The utility of an agent $i$ who is awarded $J$ units and pays $t_{i}$ is

$$
\sum_{j=1}^{J} v_{j}\left(s_{i}\right)-t_{i} .
$$

Hence, our set-up is based on private (but possibly correlated or affiliated) valuations.

\subsection{Noisy participation}

In some cases, we follow Swinkels (2001) by considering "noisy participation". There is uncertainty regarding the exact number of active bidders in the auction, as is natural in the context of a large number of bidders. This helps rule out certain types of "degenerate" equilibria, such as one in a uniform price auction with say 1000 bidders and 500 objects, where the first 500 bidders each bid the maximum value for a single object and the other bidders bid 0 . This sort of equilibrium involves weakly dominated strategies by all the bidders, and requires their exact knowledge of the number of active bidders. If there is uncertainty about the number of bidders, such strategies no longer form an equilibrium. ${ }^{14}$ Formally, this is modelled by

Assumption 3. There is a probability $\tau>0$ that any given bidder is inactive. The inactivity is independent across bidders and is independent of $X$.

14. One can instead rule out such equilibria via an equilibrium refinement that rules out weakly dominated strategies, but with a continuum of actions one has to be careful to work with closures of such strategies and the technical details for verifying equilibrium conditions can be complicated. Noisy participation is an easy and natural condition that provides the necessary refinement without any additional machinery. 
Assumption 3 can be thought of as adding an atom in the distribution of signals at $S_{i}=0$. Laws of large numbers imply that for large enough $n$ a fraction of approximately $(1-\tau)$ of the bidders will be active.

\subsection{Auctions}

We consider uniform price and discriminatory auctions. In both of these auction formats each active bidder submits $m$ bids. We denote $i$ 's bids by $b_{i} \in[0,1]^{m}$, where the $j$-th bid of bidder $i$ is denoted $b_{i j}$. We order bids so that $b_{i j}$ is non-increasing in $j$. In both auction formats, the $k_{n}$ highest bids are each awarded a unit of the good. In the discriminatory auction bidders pay the sum of their winning bids. So, if bidder $i$ wins $1 \leq J \leq m$ units, then the payment in the discriminatory auction is $\sum_{j=1}^{J} b_{i j}$. Let $B_{-i}(l)$, denote the $l$-th highest bid among active bidders other than $i$.

In a uniform price auction the winning bidders pay the same price per unit, which is the $k_{n}+1$-st highest bid. So, if bidder $i$ wins $1 \leq J \leq m$ units, then the payment in the uniform auction is $J p$, where $p$ is the $k_{n}+1$-th highest bid out of all bids. There are a number of variations on rules for uniform price auctions. Some adjust, for instance, to make sure that a bidder $i$ 's price is not dependent on his own bid. In one such variation each bidder pays the highest non-winning bid among the other agents. Our results here are unaffected by such details.

\subsection{Equilibrium}

A strategy is a measurable function $\sigma_{i}:[0,1] \rightarrow \Delta\left([0,1]^{m}\right)$. A strategy is thus a distribution over submitted bids as a function of $i$ 's information. ${ }^{15}$ In cases where there is noisy participation, bidders still formulate a strategy, but randomly some bidders' bids never make it to the auction.

We consider Bayesian equilibria of the auctions.

In some cases we refer to pure strategy equilibria, in which case we write $b_{i j}\left(s_{i}\right)$ to indicate the $j$-th bid that $i$ submits as a function of $s_{i}$.

A theorem of Jackson and Swinkels (2005) applies to provide existence of equilibria here. ${ }^{16}$

\subsection{Asymptotic efficiency and surplus extraction}

We say that a sequence of equilibria of an auction leads to approximately efficient allocations, if the average valuations of allocated objects under the specified equilibria approaches (in probability) the maximizing average of active bidders' valuations across all possible allocations of the goods. ${ }^{17}$

We say that the seller extracts the entire surplus if the seller's revenue approaches (in probability) the maximizing sum of active bidders' valuations across all possible allocations of the goods.

15. One can equivalently work with distributional strategies à la Milgrom and Weber (1985).

16. Their theorem handles the uncertain number of bidders through the reservation values. Existence of pure strategy and monotone equilibria have been established in certain cases; see Athey (2001), McAdams (2003), Reny and Zamir (2004), and Reny (2005).

17. With a fixed number of units for sale, having the average value converge to the efficient average implies that that sum converges to the efficient sum. With a proportional number of units, having the average value converge is weaker than having the sum converge. As we will show a failure of convergence of the average in some cases, the weaker definition leads to a stronger conclusion. 


\section{A FIXED NUMBER OF UNITS}

We begin by analysing the case where a fixed number of units is being sold. Our results here can be viewed both as a benchmark and as a generalization of results in the previous literature. Here, there is no asymptotic difference between the two auction formats, which in the limit leads to an efficient outcome and where the seller extracts the entire surplus.

Theorem 1. Under a fixed supply of objects and Assumptions 1-3, all sequences of equilibria of discriminatory auctions and uniform price auctions lead to approximately efficient allocations. Moreover, in all sequences of equilibria the seller extracts the entire surplus.

It is important to note that our conclusions hold for the entire equilibrium set and hence do not depend on the exact form of the equilibrium. That is, we do not rely on the equilibrium being monotone or even in pure strategies. Our conclusion follows from the competitive pressure that arises in the limit.

Note also, that this result shows the seller can extract the entire surplus here without using any reserve price and without much worry as to the choice of auction format.

One might be tempted to argue that not only do the uniform and discriminatory price auction achieve efficient allocations and full revenue extraction, but that essentially any "standard" mechanism would do the same. This however is not true. Consider for example, a "posted-price" mechanism where a price is fixed by the seller and then in the case of a surplus demand the objects are randomly rationed among buyers who indicate a desire to buy at the posted price. The allocation will generally be inefficient and the auction yields less than full revenues. Hence, for such a conjecture to hold one needs to require that the mechanism satisfy some conditions. For instance, any mechanism that yields an approximately efficient allocation will provide the seller with the entire surplus. ${ }^{18}$ Here, it suffices to have the price that a given bidder pays for a given unit be at least as high as the highest non-winning bid of the other bidders (or some fixed-order statistic of the non-winning bids) and not exceed the winning bidder's bid for the given unit. For details, see the Appendix.

\section{A PROPORTIONAL SUPPLY OF OBJECTS}

We now examine the case where the supply of objects is proportional to the number of agents, so that $k_{n}$ grows with $n$. As we shall see the choice of auction format is now quite relevant. The uniform price auction and the discriminatory auction differ both in the efficiency of the resulting allocation and the revenues that the seller can expect. We begin by showing that while the uniform price auction yields an efficient allocation, the discriminatory auction results in inefficiency even in the limit.

\subsection{Multiple-unit demands}

Let us first consider the case where agents desire up to $m$ units of the good ( $m \geq 1)$, and we will return to the special case where each agent demands at most one object $(m=1)$.

5.1.1. Efficient allocations in uniform price auctions. We first show that a uniform price auction achieves an efficient outcome in the limit. A key intuition behind this fact is that

18. Jackson and Kremer (2004) provide results on direct mechanisms under the condition that the equilibrium allocation results in "concentrated" allocations, where the goods end up in the hands of a vanishing fraction of the agents, and only those with highest signals. Here, we show that this results from the competitive pressures. 
the probability that a bidder's actions can have a substantial influence on the price goes to 0 in the limit, as stated in the following lemma.

This result and the following theorem build almost directly from Swinkels (2001). The key difference is we are not in a world of independent types. However, the basic ideas behind his results extend, as do the proofs after some modifications that are addressed in the Appendix.

Lemma 1. Consider a setting satisfying Assumptions 1-3 and any sequence of equilibria of uniform price auctions. For any $\varepsilon>0$, there exists a large enough $n$ such that conditional on any state $X$ (in the support of $G$ ) the probability that any bidder $i$ can influence the price by more than $\varepsilon$ is smaller than $\varepsilon$.

Lemma 1 is an expression of the fact that a uniform price auction is competitive in the limit. This implies that the price converges to the value of the marginal buyer and the allocation becomes efficient. Let $p_{n}^{c}$ denote this price (the $k_{n}+1$-th highest valuation in the active population), which we term the "competitive" price.

Theorem 2. Consider a setting satisfying A1-A3 and any sequence equilibria of uniform price auctions. The equilibrium allocations are asymptotically efficient and the corresponding equilibrium prices converge to the competitive prices in probability; that is, $p_{n}-p_{n}^{c} \rightarrow 0$ in probability (where $p_{n}$ is the $k_{n}+1$-th highest bid).

This theorem tells us that uniform price auctions are "well behaved" when the supply of objects is proportional, providing efficient allocations and competitive prices. We now turn to the more muddied analysis of discriminatory auctions.

5.1.2. Inefficient allocations in discriminatory auctions. The analysis of discriminatory auctions is trickier. To get some intuition as to why, note that in a sense the discriminatory auction is like an asymmetric auction (we discuss this in more detail below). For instance, with $n=m=2=k$ each bidder's high bid competes with the other bidder's low bid, and vice versa. With a uniform auction, even with this sort of asymmetry, the incentives are reasonably straightforward, as one's bid is unlikely to affect the price. However, with a discriminatory auction, one's bid always affects the price paid (if an object is won). This asymmetry means that bids are no longer monotone in value when compared across bidders. For example, a value of $1 / 2$ on a second unit corresponds to a different signal and hence information about the potential bids of others, than a value of $1 / 2$ on a first unit. This loss of monotonicity across bidders is the key reason why the discriminatory auction is inefficient (even in the limit).

To gain some intuition behind the result consider two bidders. Bidder I values the first unit at 1 and second unit at a 1/2, bidder II values the first unit at 1/2 and the second unit at a $1 / 4$. For the allocation to be efficient, we need that a bid depends only on values. Specifically, consider the second bid of bidder I and the first bid of bidder II. If these two bids differ then there is potential for inefficiency. However, this is likely to be the case as bidders I and II have a different posterior regarding the other bidders. Since there is positive correlation, bidder I expects other bidders to be more aggressive and hence will bid higher for his second unit.

To simplify the exposition we add several assumptions.

To get some handle on bidding behaviour in discriminatory auctions, we work under the familiar strict monotone likelihood ratio property (henceforth, MLRP). To simplify the exposition we also assume continuity. 
Assumption 4. $f\left(s_{i} \mid x\right)$ and $f(x)$ are continuous in $x$, and the strict MLRP holds:

$$
\frac{f\left(s_{i} \mid x\right)}{f\left(s_{i}^{\prime} \mid x\right)}>\frac{f\left(s_{i} \mid x^{\prime}\right)}{f\left(s_{i}^{\prime} \mid x^{\prime}\right)} \text { for all } s_{i}>s_{i}^{\prime} \text { and } x>x^{\prime} .
$$

Let $\bar{v}^{n}$ denote the efficient cut-off valuation under a given realization of (active) values, that is, the $k_{n}$-th highest valuation.

Let

$$
\min \left(s_{i}\right)=\sup \left\{v \mid \lim _{n} \operatorname{Prob}\left(\bar{v}^{n} \geq v \mid S_{i}=s_{i}\right)=1\right\} .
$$

So, $\min \left(s_{i}\right)$ is the minimum of the support of $\bar{v}^{n}$ under the limiting distribution conditional on $S_{i}=s_{i} \cdot{ }^{19}$ Note that $\min \left(s_{i}\right)$ will generally be above 0 even if $\bar{v}^{n}$ has full support for each $n$ and regardless of $s_{i}$. For instance, in Example 2, $\min \left(s_{i}\right)=2 \max \left[0, s_{i-1}\right] / 3+4 / 9$.

The important aspect of this definition is that a bidder seeing $s_{i}$ would expect the lowest value still getting an object in the limit under an efficient allocation to be at least $\min \left(s_{i}\right)$. In particular, this implies that if the allocation were to be efficient, then a bidder seeing a signal $s_{i}$ and having a value for one of their objects near $\min \left(s_{i}\right)$ would expect to have to bid near $\min \left(s_{i}\right)$ to get an object.

The MLRP assumption implies that $\min \left(s_{i}\right)$ is non-decreasing.

Next we assume that there is some possibility that at least somebody gets $m$ objects.

Assumption 5. In an efficient allocation, at least one type sees a positive probability of obtaining $m$ units. That is, $\min \left(s_{i}\right)<v_{m}\left(s_{i}\right)$ for some $s_{i}$. Moreover, $\min \left(s_{i}\right)$ is continuous.

Assumption 5 is essentially without loss of generality, as otherwise we can reset $m$ to simply cover units that might be obtained.

Since $\min \left(s_{i}\right)$ and $v_{m}\left(s_{i}\right)$ are continuous and $\min \left(s_{i}\right)$ lies above $v_{m}\left(s_{i}\right)$ at $s_{i}=0$, and below $v_{m}\left(s_{i}\right)$ for some $s_{i}$ under Assumption 5, it follows that there exists $s^{*}$ so that $\min \left(s^{*}\right)=v_{m}\left(s^{*}\right)$.

So $s^{*}$ identifies a signal that lets a bidder know that his or her valuation for an $m$-th object is just on the border of sometimes vs. never being awarded an object under an efficient allocation. There could be many such signals, let us pick any one of them.

We assume that

Assumption 6. $\min \left(v_{m}\left(s^{*}\right)\right)<\min \left(s^{*}\right)$, for some $s^{*}$ such that $\min \left(s^{*}\right)=v_{m}\left(s^{*}\right)$.

While Assumption 5 is fairly mild, Assumption 6 has a bit more to it. Assumption 6 requires that what an agent sees as the lowest possible valuation getting an object in the limit in an efficient allocation is increasing between $v_{m}\left(s^{*}\right)$ and $s^{*}$ (or at least comparing the endpoints). This means that signals convey some information about the support of the efficient cut-off value $\bar{v}^{n}$, at least in the limit.

The fact that signals convey information about the efficient cut-off value is sufficient to ensure that agents who have nearly the same valuation for one of their objects, but hold different signals, will bid substantially differently in equilibrium. It is easily checked that Assumption 6 is satisfied in Example 2. One can also find reasonable examples that violate Assumption 6. For example, if $X$ is uniformly distributed on $[0,1]$ and $s_{i}$ is uniformly distributed on $[X, 1]$, then $\min \left(s_{i}\right)=0.5$ for all $s_{i}$, which violates Assumption $6 .^{20}$

19. Note that the limiting distribution can have a support that varies with signals even without any of the underlying distributions having supports that vary with signals.

20. Nevertheless, we conjecture that the same result holds in situations where agents can have the same values for units (say, one for his or her first unit and the other for the $m$-th unit) but have sufficiently different beliefs about what 
Theorem 3 states that under the above assumptions, the discriminatory auction always yields inefficient outcomes even in the limit.

Theorem 3. Under Assumptions 1-6 and $m>1$, every sequence of equilibria of discriminatory auctions fails to be asymptotically efficient.

The intuition behind the theorem is similar to the intuition we discussed above and was illustrated in Example 2. If the allocation were to be efficient, some of the marginal bids would be coming from bidders who are bidding on their first unit (with value $s_{i}$ ), while others would be coming from bidders who are bidding on their last unit (with value $v_{m}\left(s_{i}\right)$ ). Under Assumption 6 , these two classes of bidders are both near the margin for an efficient allocation, but have different beliefs about the cut-off value. This provides for different bidding behaviour for the same valuations. The resulting bids are not monotonic in values, and the allocation fails to be efficient, even approximately and asymptotically.

\subsection{Comparing revenues in the two auctions}

It is natural to conjecture that the asymptotic revenue for the discriminatory auction would be no higher than that of the uniform price auction. The reason that this seems natural is that the uniform price auction leads to an asymptotically efficient allocation and the competitive price in the limit, while the discriminatory auction can lead to an inefficient allocation. Hence, one might guess that the discriminatory auction leads to a correspondingly lower price. While this is true in some cases, it is not always true.

We begin with the case of single-unit demands. Here, it turns out that there is a difference between the auctions; but somewhat surprisingly, this is true even though both auctions lead to the same efficient allocation.

\subsection{Comparing revenues with single-unit demands}

A well-studied case in the literature (e.g. see Milgrom, 1981) is one where agents demand only a single unit $(m=1)$.

Note that under Assumption 4 there exist (symmetric) equilibria for both the uniform and discriminatory auctions and that they support the same efficient allocation, where the $k_{n}$ highest signal holders obtain the objects. The unique symmetric equilibrium strategy in the uniform price auction is for bidders to use the dominant strategy of bidding their value. An extension of the equilibrium of the first price auction described in Milgrom and Weber (1982) constitutes a symmetric equilibrium in a multiple-unit discriminatory mechanism. The following theorem refers to that equilibrium and the dominant strategy equilibrium in the uniform price auction. We conjecture that these are the unique equilibria (under Assumption 3), but do not have a proof of this. $^{21}$

the cut-off price is likely to be. The (sufficiently) different beliefs about the distribution of the cut-off price would lead to two different bids for similarly valued items and would thus create inefficiencies that exist even in the limit. Assumption 6 is sufficient to guarantee this, and it allows us to bound bidders' behaviour, even in situations where we know very little about the specific structure of the equilibrium. However, Assumption 6 is not necessary, and so some weaker condition should also suffice (but possibly with a much more difficult proof).

21. These are the unique equilibria in the class of monotonic symmetric equilibria in which bidders use pure strategies, but their overall uniqueness properties are more difficult to ascertain. In terms of uniqueness, recall that the uncertain number of bidders rules out the standard asymmetric equilibria in the uniform auction case. Techniques used by McAdams $(2005 a, b)$ to deduce monotonicity and uniqueness in first price auctions might be extendable to our setting. 
Theorem 4. Let Assumptions 2 and 4 hold and $\frac{k_{n}}{n} \rightarrow b$ where $1>b>0$. The uniform price auction yields higher expected revenue per capita (and per unit) than a discriminatory price auction, by an amount that is bounded below as $n \rightarrow \infty$.

We prove the theorem (in the Appendix) using the following technique. ${ }^{22}$ Using the logic of Milgrom and Weber (1982), we can show that the expected payment of any given signal holder in a uniform auction is at least that of his clone in a discriminatory auction. We then argue that above a certain signal (one that is approximately sure to receive an object for large $n$ ) the payment schedule for a discriminatory auction flattens out, as higher signal holders can always bid as if they had this lower signal and will still be approximately certain to get an object. In the uniform auction, however, this payment schedule does not flatten out, as under strict MLRP observers of higher signals expect higher market clearing prices. Through this, we establish a bound on the difference in revenues.

While Theorem 4 shows that a uniform auction leads to higher expected revenues than a discriminatory auction when each bidder demands at most one unit, this unambiguous ranking no longer holds when there are multiple-unit demands. With multiple-unit demands the rankings can go either way.

To see that uniform auction revenues can exceed discriminatory auction revenues with multiple-unit demands, consider a simple extension of the single-unit case, where bidders value all of their objects at the same value. In that case, bidder behaviour in the limit looks similar to the single-unit case, and so uniform auctions in that situation lead to higher revenues than discriminatory auctions.

In contrast, in a situation where each bidder has a large difference between marginal valuations for some objects, then we saw in Example 4 that it is also possible for the revenue of the discriminatory auction to exceed that of the uniform price auction.

These results suggest that there might be some relationship between the shape of individual demand curves and the revenue rankings of the two auction formats. While this seems to have some intuition behind it, we have not been able to discover any such general result.

\section{DISCUSSION: ASYMMETRIES AND MULTI-DIMENSIONAL INFORMATION}

One might conjecture that in a competitive environment the particulars of the market structure is largely irrelevant. We have shown that this conclusion is limited to the case in which there is a vanishing number of objects for sale relative to the number of bidders. When the number of objects for sale is proportional to the number of bidders, the particulars of the auction format matter. While the efficiency properties of some prominent auction formats are unambiguous (but different), their relative revenue ranking depends on the circumstances.

Our set-up may be viewed as somewhat restrictive as information is one-dimensional and bidders have their information drawn from the same distribution. However, it is important to emphasize that generalizing the model to allow for asymmetries and multi-dimensional information would not change our main conclusions. With many bidders the uniform price auction would still yield the efficient outcome, as agents would bid according to their values. ${ }^{23}$ A discriminatory auction with proportional supply would still yield inefficient outcomes for the same reasons that we saw here. In fact, the intuition behind our result is precisely that of asymmetries among

22. This technique can also be used to establish a parallel result for the case of common values (see Jackson and Kremer, 2006), and in fact the result extends to situations where bidders' valuations depend in monotone ways on both their signal and the common factor $X$.

23. It is important that the values be private for this conclusion. With interdependent valuations, attaining efficiency is problematic as shown quite powerfully by Jehiel and Moldovanu $(2001 a, b)$. 
bidders: bidders with the same values for some objects can bid differently as their information about the likely bidding behaviour of other agents can differ. Hence, in a sense the fact that we assume a symmetric set-up with uni-dimensional signals makes the results more striking, as interim asymmetries with uni-dimensional signals are already enough to produce significant differences between the auction formats. Incorporating asymmetries or multi-dimensional information would simply amplify the differences between the auction formats.

\section{APPENDIX}

\section{Details behind Example 3}

Let $F_{Y_{n / 2} \mid V_{i}}$ and $f_{Y_{n / 2} \mid V_{i}}$ denote the distribution and density functions of the median signal conditional on $V_{i}$. A similar argument to that of Milgrom and Weber (1982) shows that a monotonic symmetric pure strategy equilibrium bidding function in the $n$-th economy, $b^{n}$, is the solution to the differential equation

$$
b^{n \prime}\left(v_{i}\right) F_{Y_{n / 2} \mid v_{i}}\left(v_{i} \mid v_{i}\right)=f_{Y_{n / 2} \mid v_{i}}\left(v_{i} \mid v_{i}\right)\left(v_{i}-b^{n}\left(v_{i}\right)\right)
$$

with a boundary condition $b^{n}(0)=0$. Note that $F_{Y_{n / 2} \mid v_{i}}\left(v_{i} \mid v_{i}\right) \rightarrow \frac{\ln (2)}{\ln \left(1 / v_{i}\right)}$ (which can be verified by noting that $F_{Y_{n / 2} \mid v_{i}}\left(v_{i} \mid v_{i}\right)=F_{X \mid v_{i}}\left(2 v_{i} \mid v_{i}\right)$, and then calculating $\left.F_{X \mid v_{i}}\right)$, while $f_{Y_{n / 2} \mid v_{i}}\left(v_{i} \mid v_{i}\right) \rightarrow \frac{1}{v_{i} \ln \left(1 / v_{i}\right)}$ if $v_{i}<0 \cdot 5$ and $\rightarrow 0$ if $v_{i}>0 \cdot 5$. Hence,

$$
v_{i} b^{\prime}\left(v_{i}\right) \ln (2)=v_{i}-b^{n}\left(v_{i}\right)
$$

for $v_{i}<0 \cdot 5$ and $b^{\prime}\left(v_{i}\right)=0$ for $v_{i}>0 \cdot 5$. Together with the boundary condition, $b(0)=0$, this implies that

$$
b(v)= \begin{cases}\frac{1}{1+\ln (2)} v & \text { for } v<0.5 \\ \frac{1}{1+\ln (2)} 0.5 & \text { for } v \geq 0.5\end{cases}
$$

Proof of Theorem 1. Consider a fixed supply of objects such that $k_{n}=k$, and a setting that satisfies Assumptions $1-3$. We prove the claim by relying on the following properties that are satisfied by both auctions:

- Both auctions award objects to the highest bids.

- In both auctions the price that a given bidder pays for a given unit is at least as high as the highest non-winning bid of the other bidders and does not exceed the given bidder's bid for the given unit.

- In both auctions the price that a given bidder pays is non-decreasing in the vector of own bids.

We argue that in any auction that satisfies the above properties all sequences of equilibria lead to approximately efficient allocations and are such that the seller extracts the entire surplus.

To see this let $p_{n}$ denote the lowest price paid for a unit in auction $n$. We prove the theorem by showing that there is a vanishing probability that a bidder finds the lowest equilibrium price, $p_{n}$, to be significantly lower than his value for the first unit. In particular, we show that for any $i, \delta>0$ and large enough $n$,

$$
\operatorname{Pr}\left(p_{n}<v_{1}\left(S_{i}\right)-\delta\right)=\operatorname{Pr}\left(p_{n}<S_{i}-\delta\right) \leq \delta
$$

Under Assumption $1, S_{i}$ has a non-vanishing probability of being within $\delta / 2$ of the highest possible signal. Hence, the distance between $p_{n}$ and the highest realized signal converges to 0 in probability, which implies full revenue extraction. This implies the theorem as given the private values setting (Assumption 2) and the structure of the auctions, bidders never expect to pay more than their value in equilibrium (as they would do better by lowering their bids otherwise). Hence, we also get approximate efficiency, as the objects must be allocated to agents with valuations between $1-\delta^{\prime}$ and the highest signal, for arbitrary $\delta^{\prime}$. So let us show (A.1). Note that since the distribution is symmetric it is enough to show that (A.1) holds for a sequence $\left\{i_{n}\right\}$. This is useful, since the equilibrium need not be symmetric. Let $\varepsilon_{n}$ converge to 0 more slowly than $\frac{1}{n}$; that is, $\frac{1}{n \varepsilon_{n}} \rightarrow 0$. Let $A_{i}^{n}$ denote the set of signals for which bidder $i$ 's conditional expected number of objects won in the auction is less than $\varepsilon_{n}$. That is, letting $q_{i}^{n}$ be the random variable representing the number of objects that bidder $i$ obtains in the $n$-th auction,

$$
A_{i}^{n}=\left\{s \mid E\left(q_{i}^{n} \mid S_{i}=s\right)<\varepsilon_{n}\right\}
$$

Note that in the definition of $A_{i}^{n}$ we integrate only on the events in which $i$ participates. By the definition of $A_{i}^{n}$, there must exist a sequence of $\left\{i_{n}\right\}$ for which $\operatorname{Pr}\left(S_{i_{n}} \in A_{i_{n}}^{n}\right) \rightarrow 1$, as otherwise, the total number of objects expected to 
be received would exceed the total supply (recalling that $\varepsilon_{n}$ converges to 0 at a slower rate than $1 / n$ ). Suppose that for some $\delta$ the $k$-th highest bid offered by bidders other than $i_{n}, B_{-i_{n}}(k)$, satisfies

$$
\operatorname{Pr}\left(B_{-i_{n}}(k)<s-\delta \mid S_{i_{n}}=s\right)>\delta
$$

for infinitely many $n$ and some $s \in A_{i_{n}}^{n}$ for each of those $n$. In that case, for large enough $n, i_{n}$ could deviate from the purported equilibrium strategy to strictly gain by bidding $s-\delta$, which would be a contradiction. Thus, for any $\delta$, for large enough $n$,

$$
\operatorname{Pr}\left(B_{-i_{n}}(k)<s-\delta \mid S_{i_{n}}=s\right) \leq \delta
$$

for all $s \in A_{i_{n}}^{n}$. This is not sufficient to conclude the proof as in the uniform price auction the price equals the $k+1$-th highest bid and one needs to worry about the possibility of equilibria with minimal prices. Nevertheless, Lemma A1 (below) shows that under noisy participation (Assumption 3), inequality (A.2) implies that for any $\delta$, for large enough $n$

$$
\operatorname{Pr}\left(B_{-i_{n}}(k+1)<s-\delta \mid S_{i_{n}}=s\right) \leq \delta
$$

for any $s \in A_{i_{n}}^{n}$. We also know from the structure of the auction that $p_{n} \geq B_{-i}(k+1)$ for any $i$. Thus, it follows from (A.3) that for any $\delta$ and large enough $n$

$$
\operatorname{Pr}\left(p_{n}<s-\delta \mid S_{i_{n}}=s\right) \leq \delta
$$

for all $s \in A_{i_{n}}^{n}$. Integrating (A.4) over $s \in A_{i_{n}}^{n}$ given that $\operatorname{Pr}\left(S_{i_{n}} \in A_{i_{n}}^{n}\right) \rightarrow 1$ implies that for any $\delta, \operatorname{Pr}\left(p_{n}<S_{i_{n}}-\delta\right) \leq 2 \delta$, for large enough $n$ (where the extra $\delta$ accounts for the vanishing probability of signals outside of $A_{i_{n}}^{n}$ ), which implies the desired claim. $\|$

We now prove Lemma A1, which was referred to in the proof above.

Lemma A 1. Under noisy participation (Assumption 3) and a fixed number of units $k_{n}=k$ for sale if, for given sequences of bidders $\left\{i_{n}\right\}$ and signals $\left\{s_{n}\right\}$ that are such that for any given $\delta>0$

$$
\operatorname{Pr}\left(B_{-i_{n}}(k)<s_{n}-\delta \mid S_{i_{n}}=s_{n}\right) \leq \delta
$$

for large $n$, then also for any $\delta>0$ for large enough $n$

$$
\operatorname{Pr}\left(B_{-i_{n}}(k+1)<s_{n}-\delta \mid S_{i_{n}}=s_{n}\right) \leq \delta .
$$

Proof. Suppose the contrary, then we can find some $\delta^{*}>0$ so that

$$
\operatorname{Pr}\left(B_{-i}(k+1)<s_{n}-\delta^{*} \mid S_{i_{n}}=s_{n}\right)>\delta^{*}
$$

despite the fact that

$$
\operatorname{Pr}\left(B_{-i}(k)<s_{n}-\delta^{*} \mid S_{i_{n}}=s_{n}\right) \rightarrow 0 .
$$

Using our version of the "Mineral Rights" model we can find a sequence of states in the support of the set of states, $\left\{x_{n}\right\}$, so that

$$
\operatorname{Pr}\left(B_{-i}(k+1)<s_{n}-\delta^{*} \mid X=x_{n}\right)>\delta^{\prime}
$$

for some $\delta^{\prime}>0$ despite the fact that

$$
\operatorname{Pr}\left(B_{-i}(k)<s_{n}-\delta^{*} \mid X=x_{n}\right) \rightarrow 0 .
$$

For every bidder $j \neq i_{n}$ let $Z_{j}^{n}$ be the (random) number of bids that $j \neq i_{n}$ has above $s_{n}-\delta$; so $Z_{j}^{n} \in\{0,1, \ldots, m\}$. To account for how many bids by $j \neq i_{n}$ end up above $s_{n}-\delta>0$ under the participation noise, Assumption 3, we define a new random variable, which is equal to $Z_{j}^{n}$ with probability $(1-\tau)$ and equals 0 with probability $\tau$. Let the resulting random variables be denoted as $\hat{Z}_{j}^{n}$ which, conditional on $X=x_{n}$, are independent (but not identically distributed). In this notation, (A.8) and (A.9) (applying (A.9) at $s_{n}$ ) become

$$
\operatorname{Pr}\left(\sum_{j \neq i_{n}} \hat{Z}_{j}^{n}<k+1 \mid X=x_{n}\right)>\delta^{\prime}
$$

and

$$
\operatorname{Pr}\left(\sum_{j \neq i_{n}} \hat{Z}_{j}^{n}<k \mid X=x_{n}\right) \rightarrow 0 .
$$


By subtracting (A.11) from (A.10), it follows that

$$
\operatorname{Pr}\left(\sum_{j \neq i_{n}} \hat{Z}_{j}^{n}=k \mid X=x_{n}\right)>\delta^{\prime}
$$

for infinitely many $n$.

We get a contradiction by arguing that (A.12) implies that $\operatorname{Pr}\left(\sum_{j \neq i_{n}} \hat{Z}_{j}^{n}<k \mid X=x_{n}\right)$ is above some level for infinitely many $n$.

To see this write,

$$
\operatorname{Pr}\left(\sum_{j \neq i_{n}} \hat{Z}_{j}^{n}=k \mid X=x_{n}\right)=\sum_{z} \operatorname{Pr}\left(\sum_{j \neq i_{n}} \hat{Z}_{j}^{n}=k \mid \sum_{j \neq i_{n}} Z_{j}^{n}=z\right) \operatorname{Pr}\left(\sum_{j \neq i_{n}} Z_{j}^{n}=z \mid X=x_{n}\right) .
$$

Define the set $A_{n}$ by

$$
A_{n}=\left\{z \mid \operatorname{Pr}\left(\sum_{j \neq i_{n}} \hat{Z}_{j}^{n}=k \mid \sum_{j \neq i_{n}} Z_{j}^{n}=z\right) \geq \delta^{\prime} / 4\right\} .
$$

From (A.12) and (A.13), it follows that $\operatorname{Pr}\left(A_{n} \mid X=x_{n}\right) \geq \delta^{\prime} / 4$. Note that $\operatorname{Pr}\left(\sum_{j} \hat{Z}_{j}^{n}=k \mid \sum_{j \neq i_{n}} Z_{j}^{n}=z\right)$ goes to 0 as $z$ gets large (as it is bounded above by the probability of getting at most $k 1$ 's realized out of $N$ Bernoulli random variables with a probability of $(1-\tau)$ on each). Thus, there exists a bound $M$ so that for all large enough $n$, if $z \in A_{n}$ then $z<M$. However, this implies that for each $z \in A_{n}$

$$
\operatorname{Pr}\left(\sum_{j \neq i_{n}} \hat{Z}_{j}^{n}<k \mid \sum_{j \neq i_{n}} Z_{j}^{n}=z\right) \geq \operatorname{Pr}\left(\sum_{j \neq i_{n}} \hat{Z}_{j}^{n}=0 \mid \sum_{j \neq i_{n}} Z_{j}^{n}=z\right)>\tau^{M} \cdot{ }^{24}
$$

Since $\operatorname{Pr}\left(A_{n} \mid X=x_{n}\right) \geq \delta^{\prime} / 4$, we have shown that

$$
\operatorname{Pr}\left(\sum_{j \neq i_{n}} \hat{Z}_{j}^{n}<k \mid X=x_{n}\right) \geq \tau^{M} \delta^{\prime} / 4
$$

for infinitely many $n$, which contradicts (A.11). $\quad \|$

Proof of Lemma 1. We first measure the influence a bidder has on prices by looking at the event in which he is able to push the price above some threshold $y \in[0,1]$, given that the price would be below $y$ in the absence of the bidder's bids. For bidder $i$ to be able to push the price above $y$ it must be that $B_{-i}\left(k_{n}-m\right)>y$ and $B_{-i}\left(k_{n}\right)<y$. Laws of large numbers imply that this happens with low probability. Specifically, the argument used in Lemma 9.2 in Swinkels (2001) shows that for any $\varepsilon>0$ there exists some $N_{\varepsilon}$ so that $n>N_{\varepsilon}$ implies that for any state $X$ and bidder $i$ :

$$
\operatorname{Pr}\left(B_{-i}\left(k_{n}-m\right)>y \text { and } B_{-i}\left(k_{n}\right)<y \mid X\right)<\varepsilon .
$$

A sketch of the argument is as follows. There are $(n-1) m$ bids of bidders besides $i$. One can define random variables $\left\{Z_{j}\right\}_{j \neq i}$ that give the number of bids above $y$ that are submitted by bidder $j$. The probability that $i$ pushes the price above $y$ when starting below is then bounded by $\operatorname{Pr}\left(\sum_{j \neq i} Z_{j} \in\left[k_{n}-m, k_{n}\right]\right)$. Conditional on $X$ bids are independent, and hence so are the $\left\{Z_{j}\right\}$. This implies that this probability is negligible for large $n$ since $m$ is finite.

The above inequality implies that if we fix a positive integer $J$, then for any $j \in\{1, \ldots, J\}$ there exists $N_{j}$ such that for $n>N_{j}$

$$
\operatorname{Pr}\left(B_{-i}\left(k_{n}-m\right)>\frac{j}{J} \text { and } B_{-i}\left(k_{n} t\right)<\frac{j}{J} \mid X\right)<\frac{1}{J^{2}} .
$$

Letting $N^{*}=\max _{j} N_{j}$, it follows that for all $n>N^{*}$

$$
\operatorname{Pr}\left(\exists j: B_{-i}\left(k_{n}-m\right)>\frac{j}{J} \text { and } B_{-i}\left(k_{n}\right)<\frac{j}{J} \mid X\right)<\frac{J}{J^{2}}=\frac{1}{J}
$$

24. One could multiply the inequality by the number of different ways of choosing $M$ alternatives out of $\dot{n}$. However, since the expression would not be monotone decreasing in $M$ we use a more modest bound. 
Let $p_{1}$ denote the price if bidder $i$ submits the maximal possible bid on all of his units, and let $p_{0}$ denote the price if $i$ bids 0 on all of his units. The above inequality implies that for any $J$ there exists $N_{J}$ such that $\operatorname{Pr}\left(p_{1}-p_{0}>\frac{2}{J}\right)<\frac{1}{J}$ for $n>N_{J}$. To conclude the proof let $J=2 / \varepsilon$.

Proof of Theorem 2. First, let us show that the price converges to the competitive price. That is, for any $\delta>0$, for all high enough $n, \operatorname{Pr}\left(\left|p_{n}^{c}-p_{n}\right|>\delta\right)<\delta$.

Suppose that this is not the case, so that there exists $\delta>0$ such that for all $n$ (taking a subsequence if necessary), $\operatorname{Pr}\left(p_{n}^{c}-p_{n}>\delta\right)>\delta$. [The case where $p_{n}$ exceeds $p_{n}^{c}$ is analogous.] Since both prices and values are bounded in [0,1] there exists some $\delta^{*}>0$ and some $a \in[0,1]$ such that for all $n^{25}$

$$
\operatorname{Pr}\left(p_{n}<a \text { and } p_{n}^{c}>a+\delta^{*}\right)>\delta^{*} .
$$

Hence, for large enough $n$ there is a probability bounded away from 0 that some bidder who values a unit above $a+\frac{\delta^{*}}{2}$ does not obtain that object and the price is less than $a$. Consider a deviation for such bidders to bid truthfully instead. This increases the expected number of units that such a bidder gets, but may also affect the clearing price. However, by Lemma 1 the price implications for such a deviation are negligible for large $n$. This yields a contradiction as it guarantees an extra unit at a profit bounded away from 0 for such a bidder for large enough $n$, with negligible price impact.

Next, note that a similar argument to that above (again invoking Lemma 1) implies that although bidders may place some bids above or below their corresponding values in equilibrium, this can only be in cases where for large enough $n$, changing those bids to be equal to the corresponding values would have a negligible probability of affecting the equilibrium allocation.

The approximate efficiency follows from the convergence of price to the competitive one, and bidders bidding as if they bid their values. $\|$

Proof of Theorem 3. The following lemma is useful.

Consider an $n$-bidder discriminatory auction. Let $p_{n}$ denote the minimal price paid in equilibrium, that is, the $k_{n}$-th highest bid, and let $b_{i j}^{n}\left(s_{i}\right)$ denote the bid by $i$ in the $n$-th auction for a $j$-th object when observing $s_{i}$. Also, for any $1 \leq j \leq m$ and $\delta>0$ let

$$
\begin{aligned}
& A_{i, j, \delta}^{n}=\left\{s_{i} \mid \operatorname{Pr}\left(v_{j}\left(s_{i}\right)>\bar{v}^{n}+\delta \quad \text { and } \quad p_{n}>b_{i j}^{n}\left(s_{i}\right)\right)>\delta\right\}, \\
& C_{i, j, \delta}^{n}=\left\{s_{i} \mid \operatorname{Pr}\left(v_{j}\left(s_{i}\right)<\bar{v}^{n}-\delta \quad \text { and } \quad p_{n}<b_{i j}^{n}\left(s_{i}\right)\right)>\delta\right\} .
\end{aligned}
$$

Lemma A2. If there exists $\delta>0$ such that for all bidders $i$, all large enough $n$, and some $j$ either $\operatorname{Pr}\left(A_{i, j, \delta}^{n}\right)>\delta$ or $\operatorname{Pr}\left(C_{i, j, \delta}^{n}\right)>\delta$, then the discriminatory price auction is not asymptotically efficient. That is, the expected efficiency loss per capita does not converge to 0 .

Proof. Suppose that $\operatorname{Pr}\left(A_{i, j, \delta}^{n}\right)>\delta$ for arbitrary large $n$; the case where $\operatorname{Pr}\left(C_{i, j, \delta}^{n}\right)>\delta$ is similar. Any signal that belongs to this set results in an expected efficiency loss of at least $\delta^{2}$. The fact that $\operatorname{Pr}\left(A_{i, j, \delta}^{n}\right)>\delta$ implies (appealing to the law of large numbers) that there is an expected ex ante efficiency loss that is bounded away from 0 when summing across bidders.

Assume by contradiction that the outcome is asymptotically efficient.

First, let us argue that there exists $\delta_{1}$ such that $b_{i 1}^{n}\left(s_{i}\right)<v_{m}\left(s^{*}\right)-\delta_{1}$ for all bidders $i$ and all signals $s_{i} \in\left[v_{m}\left(s^{*}\right), v_{m}\left(s^{*}\right)+\delta_{1}\right]$ for large enough $n$. Using the continuity of $\min (s)$ and Assumptions 5 and 6 we conclude that there exists an $\varepsilon$ such that agents with a signal $s_{i} \in\left[v_{m}\left(s^{*}\right), v_{m}\left(s^{*}\right)+\varepsilon\right]$ can expect to win their first unit with a probability of at least $\varepsilon$ by bidding $v_{m}\left(s^{*}\right)-\varepsilon$ for large enough $n$. This follows as prices never exceed $\bar{v}^{n}$. In equilibrium, these bidders make an expected profit on their first unit of at least $\varepsilon^{2}$ and hence they must be bidding below their value by at least $\varepsilon^{2}$. If we let $\delta_{1}=\varepsilon^{2}$ we get that $b_{1}^{n}\left(s_{i}\right)<v_{m}\left(s^{*}\right)-\delta_{1}$ for all signals $s_{i} \in\left[v_{m}\left(s^{*}\right), v_{m}\left(s^{*}\right)+\delta_{1}\right]$.

Next, note that there exists some $\delta_{2}>0$ so that $s_{i} \in\left[s^{*}, s^{*}+\delta_{2}\right]$ implies that $b_{i, m}^{n}\left(s_{i}\right)>v_{m}\left(s^{*}\right)-\delta_{1}$. This follows since bidders with signals close to (but above) $s^{*}$ have a low but positive probability of winning their $m$-th object in an efficient allocation, but that probability goes to 0 in $n$ if they underbid by any fixed amount. As a result they bid close to their reservation value on their $m$-th object for large $n$. Hence, there exists some $\delta_{2}>0$ so that $s_{i} \in\left[s^{*}, s^{*}+\delta_{2}\right]$ implies that $b_{m}^{n}\left(s_{i}\right)>v_{m}\left(s^{*}\right)-\delta_{1}$.

25. Partition [0,1] into $2 / \delta$ intervals of size $\delta / 2$ each, $\left\{\left[a_{i}, a_{i+1}\right]\right\}_{i=1}^{1 / 2 \delta}$. The case in which $p^{n}<p_{n}^{c}-\delta$ implies that we can find some $a_{i}$ so that $p_{n}<a_{i}$ and $p_{n}^{c}>a_{i+1}$. This implies that there exists some interval for which $\operatorname{Pr}\left(p_{n}<\right.$ $a_{i}$ and $\left.p_{n}^{c}>a_{i+1}\right)>\delta^{2} / 2$. Hence, we let $\delta^{*}=\delta^{2} / 2$. 
If we let $\delta^{*}=\min \left(\delta_{1}, \delta_{2}\right)$ we conclude that agents with signal $s_{i} \in\left[v_{m}\left(s^{*}\right), v_{m}\left(s^{*}\right)+\delta_{1}\right]$ bid on their first unit no more than $v_{m}\left(s^{*}\right)-\delta^{*}$ while agents with signal $s_{i} \in\left[s^{*}, s^{*}+\delta^{*}\right]$ bid on their $m$-th unit at least this amount. Consider now the event that

$$
\bar{v}^{n} \in\left(v_{m}\left(s^{*}\right)+2 \delta^{*} / 5, v_{m}\left(s^{*}\right)+3 \delta^{*} / 5\right) .
$$

Since we assume that the distribution of signals has a full support, this event occurs with some positive probability $\delta^{* *}$.

Consider two cases:

(i) $p_{n} \geq v_{m}\left(s^{*}\right)-\delta^{*}$ : This event maps to those of type A in Lemma A2. Bidders with types $s_{i} \in\left[v_{m}\left(s^{*}\right)+4 \delta^{*} / 5\right.$, $\left.v_{m}\left(s^{*}\right)+\delta^{*}\right]$ are not awarded their first object despite the fact that their valuation exceeds the cut-off by more than $\delta^{*} / 5$.

(ii) $p_{n}<v_{m}\left(s^{*}\right)-\delta^{*}$ : This event maps to those of type $\mathrm{C}$ in Lemma A2. Bidders with types $s_{i} \in\left[v_{m}\left(s^{*}\right), v_{m}\left(s^{*}\right)+\right.$ $\left.\delta^{*} / 5\right]$ are awarded their $m$-th unit despite the fact that their valuation is lower than the cut-off by more than $\delta^{*} / 5$.

Since either (i) or (ii) occurs with probability of at least $\delta^{* *} / 2$ for large enough $n$, Lemma A2 implies a contradiction.

Proof of Theorem 4. Let $\bar{t}^{n, d}\left(s_{i}\right), \bar{t}^{n, u}\left(s_{i}\right)$ be the expected payment (unconditional on whether a bidder wins) of a bidder conditional on observing signal $S_{i}=s_{i}$, in the discriminatory and the uniform price mechanisms, respectively. The expected revenues in the respective mechanisms are

$$
n \int \bar{t}^{n, \ell}\left(s_{i}\right) d F\left(s_{i}\right) \quad \text { for } \ell=u, d .
$$

Given Assumptions 2 and 4, an argument similar to that underlying Theorem 15 in Milgrom and Weber (1982) implies that

$$
\bar{t}^{n, u}\left(s_{i}\right) \geq \bar{t}^{n, d}\left(s_{i}\right) \text { for every } n \text { and } s_{i} .
$$

This implies that the expected revenue in the uniform price mechanism is no lower than in the discriminatory one. However, it does not guarantee that there exists a positive difference that is bounded below as we increase the number of bidders. To show that such gap exists, we argue that there is an interval in which $\bar{t}^{n, d}\left(s_{i}\right)$ becomes flat as $n$ increases and that on the same interval, $\bar{t}^{n, u}\left(s_{i}\right)$ is increasing.

We identify the interval as follows. Let $s^{*}(x)$ be the solution to $F\left(s_{i} \mid x\right)=1-b$, where recall $\frac{k_{n}}{n} \rightarrow b$. Note that under Assumption $4, s^{*}(x)$ is increasing and continuous in $x$. Let the interval be $\left[s^{*}, 1\right]$, where $s^{*}$ is the maximum of the support of the random variable $s^{*}(X)$. So, in the limit in an efficient allocation, any value above $s^{*}$ should be getting an object, regardless of the realization of $X$.

Let us first show that $\bar{t}^{n, d}\left(s_{i}\right)$ becomes flat as $n$ increases on the interval $\left[s^{*}, 1\right]$. Note that $s^{*}<1$ since the distribution of $S_{i}$ conditional on $X=1$ is described by a density function and $b<1$, and so $1>s^{*}(1) \geq s^{*}$. Also, since $f\left(s_{i}\right)>0$ for all $s_{i}$, there is a positive (unconditional) probability that $S_{i} \in\left[s^{*}, 1\right]$. For any $s_{i} \in\left(s^{*}, 1\right]$, there is a large enough $n$ so that a bidder observing $S_{i}=s_{i}$ has an arbitrarily high (pre-specified) probability of observing one of the highest $k_{n}$ signals. This implies that an agent who has a signal $s_{i}>s^{*}$ can pretend to have a lower signal with a minimal effect on the probability of getting an object, for some large enough $n$. Hence, we conclude that for any $s_{i}>s_{i}^{\prime}>s^{*}$

$$
\bar{t}^{n, d}\left(s_{i}\right)-\bar{t}^{n, d}\left(s_{i}^{\prime}\right) \rightarrow 0 .
$$

This implies that while $\bar{t}^{n, d}\left(s_{i}\right)$ is increasing in $s_{i},{ }^{26}$ it converges to being flat over the interval $\left(s^{*}, 1\right]$ as $n$ increases.

We now complete the proof of the theorem by showing that indeed $\bar{t}^{n, u}$ converges point-wise on $\left(s^{*}, 1\right]$ to a function $t^{*}$ that is increasing over $\left(s^{*}, 1\right]$. We first note with private values bidders bid their values. That is, a bidder who values the good at $s_{i}$ bids $s_{i}$. This implies that the price is given by the $k_{n+1}$ highest signal. Hence, if $s_{i}>s^{*}$, since agent $i$ wins the auction with certainty it follows that:

$$
\bar{t}^{n, u}\left(s_{i}\right) \rightarrow E\left(s^{*}(X) \mid S_{i}=s_{i}\right)
$$

To see that indeed $\bar{t}^{*}\left(s_{i}\right)$ is increasing on $\left(s^{*}, 1\right]$ note first $s^{*}(x)$ is increasing in $x$. Moreover, Assumption 4 implies that the distribution of $X$ conditional on $S_{i}=s_{i}$ is stochastically dominated by the distribution of $X$ conditional on $S_{i}=s_{i}^{\prime}$, where $s_{i}^{\prime}>s_{i}$. The result then follows from the stochastic dominance.

Acknowledgements. We are grateful for financial support from the Guggenheim Foundation, the Center for Advanced Studies in Behavioral Sciences, and the National Science Foundation under grants SES-9986190 and SES-0316493. We thank Jeremy Bulow, Laurent Mathevet, Tom Palfrey, and Jeroen Swinkels for helpful conversations and suggestions on earlier drafts, as well as Juuso Välimäki and two anonymous referees.

26. This follows from incentive compatibility (Assumption 2) and the strict MLRP (Assumption 4). 


\section{REFERENCES}

ATHEY, S. (2001), "Single Crossing Properties and the Existence of Pure Strategy Equilibria in Games of Incomplete Information", Econometrica, 69, 861-890.

AUSUBEL, L. and CRAMTON, P. (2002), "Demand Reduction and Inefficiency in Multi-Unit Auctions" (Mimeo, University of Maryland).

BACK, K. and ZENDER, J. (1993), "Auctions of Divisible Goods: On the Rationale for the Treasury Experiment”, Review of Financial Studies, 6 (4), 733-764.

BALI, V. and JACKSON, M. O. (2002), "Asymptotic Revenue Equivalence”, Journal of Economic Theory, 106, 161-176.

BIKHCHANDANI, S. and HUANG, C.-F. (1989), "Auctions with Resale Markets: An Exploratory Model of Treasury Bill Markets", The Review of Financial Studies, 2 (3), 311-339.

BULOW, J. and KLEMPERER, P. (1996), "Auctions Versus Negotiations", The American Economic Review, 86 (1), 180-194.

CHARI, V.-V. and WEBER, R. J. (1992), "How the U.S. Treasury Should Auction its Debt", Quarterly Review of the Federal Reserve Bank of Minneapolis, 16 (4), 3-12.

FRIEDMAN, M. (1960) A Program for Monetary Stability (New York: Fordham University Press).

GOEREE, J. K. and OFFERMAN, T. (1999), "Competitive Bidding in Auctions with Private and Common Values" (Mimeo, University of Virginia).

HORTASÇU, A. (2001), "Mechanism Choice and Strategic Bidding in Divisible Good Auctions: An Empirical Analysis of the Turkish Treasury Auction Markets" (Mimeo, University of Chicago).

JACKSON, M. O. and KREMER, I. (2004), "The Relationship Between the Allocation of Goods and a Seller's Revenue" (Mimeo, Caltech and Stanford University), Journal of Mathematical Economics, 40, 371-392.

JACKSON, M. O. and KREMER, I. (2006), "On the Informational Inefficiency of Discriminatory Auctions", Journal of Economic Theory (in press).

JACKSON, M. O. and SWINKELS, J. M. (2005), "Existence of Equilibria in Single and Double Auctions", Econometrica, 73 (1), 93-139.

JEHIEL, P. and MOLDOVANU, B. (2001a), "A Note on Revenue Maximization and Efficiency in Multi-Object Auctions", Economics Bulletin, 3, 1-5.

JEHIEL, P. and MOLDOVANU, B. (2001b), "Efficient Design with Interdependent Valuations", Econometrica, 69, $1237-1260$

KLEMPERER, P. (2003), "Why Every Economist Should Learn Some Auction Theory", in M. Dewatripont, L. Hansen and S. Turnovsky (eds.) Advances in Economics and Econometrics: Invited Lectures to 8th World Congress of the Econometric Society (Cambridge: Cambridge University Press).

KREMER, I. (2002), "Information Aggregation in Common Value Auctions", Econometrica, 70, 1675-1682.

MASKIN, E. and RILEY, J. (2000), "Asymmetric Auctions", Review of Economics Studies, 67, 413-438.

MCADAMS, D. (2003), "Isotone Equilibrium in Games of Incomplete Information", Econometrica, 71 (4), 1191-1214.

MCADAMS, D. (2005a), "Characterizing Equilibria in Asymmetric First-Price Auctions" (Mimeo: MIT).

MCADAMS, D. (2005b), "Uniqueness in First-Price Auctions with Affiliation” (Mimeo: MIT).

MILGROM, P. (1981), "Rational Expectations, Information Acquisition, and Competitive Bidding", Econometrica, 49, 921-944.

MILGROM, P. and WEBER, R. (1982), “A Theory of Auctions and Competitive Bidding”, Econometrica, 50, $1089-1122$.

MILGROM, P. and WEBER, R. J. (1985), “Distributional Strategies for Games with Incomplete Information”, Mathematics of Operations Research, 10, 619-632.

NYBORG, K. and SUNDARESAN, S. (1996), "Discriminatory Versus Uniform Treasury Auctions: Evidence from When-Issued Transactions", Journal of Financial Economics, 42, 63-104.

RENY, P. J. (2005), “On the Existence of Monotone Pure Strategy Equilibria in Bayesian Games” (Mimeo, University of Chicago).

RENY, P. J. and ZAMIR, S. (2004), "On the Existence of Monotone Pure Strategy Equilibria Asymmetric in Asymmetric First-Price Auctions", Econometrica, 72, 1105-1126.

SWINKELS, J. (1999), "Asymptotic Efficiency for Discriminatory Private Value Auctions", Review of Economic Studies, 66, 509-528.

SWINKELS, J. (2001), "Efficiency of Large Private Value Auctions", Econometrica, 69, 37-68. 